OPEN ACCESS

Edited by:

Jee-Young Lee,

Seoul Metropolitan Government Seoul National University Boramae

Medical Center, South Korea

Reviewed by:

Sanghee Moon,

Ithaca College, United States

Chaewon Shin,

Chungnam National University,

South Korea

*Correspondence:

Inga Liepelt-Scarfone

inga.liepelt@uni-tuebingen.de

Specialty section: This article was submitted to

Parkinson's Disease and Aging-related Movement

Disorders,

a section of the journa

Frontiers in Aging Neuroscience

Received: 18 December 2021

Accepted: 21 January 2022

Published: 11 February 2022

Citation:

Becker S, Solbrig S, Michaelis K,

Faust B, Brockmann K and Liepelt-Scarfone I (2022) Divergence Between Informant and Self-Ratings

of Activities of Daily Living

Impairments in Parkinson's Disease.

Front. Aging Neurosci. 14:838674.

doi: 10.3389/fnagi.2022.838674

\section{Divergence Between Informant and Self-Ratings of Activities of Daily Living Impairments in Parkinson's Disease}

\author{
Sara Becker ${ }^{1,2}$, Susanne Solbrig ${ }^{1,3}$, Katja Michaelis ${ }^{1,3}$, Bettina Faust ${ }^{1,3}$, \\ Kathrin Brockmann ${ }^{1,3}$ and Inga Liepelt-Scarfone ${ }^{1,3,4 *}$
}

1 Department of Neurodegenerative Diseases, Hertie Institute for Clinical Brain Research, University of Tübingen, Tübingen, Germany, ${ }^{2}$ Department of Psychology, Faculty of Arts, University of Calgary, Calgary, AB, Canada, ${ }^{3}$ German Center for Neurodegenerative Diseases (DZNE), University of Tübingen, Tübingen, Germany, ${ }^{4}$ Studienzentrum Stuttgart, IB Hochschule für Gesundheit und Soziales, Stuttgart, Germany

Objective: To examine the agreement between self- and informant-reported activities of daily living (ADL) deficits in Parkinson's Disease (PD) patients, and to examine factors influencing $A D L$ ratings.

Background: In PD, the loss of functional independence is an important outcome of disease progression. The valid assessment of ADL function in PD is essential, but it is unclear to what extent informants' and patients' perceptions of their daily functions concur, and how other factors may influence both ratings.

Methods: Data of 150 PD patients who underwent cognitive and motor testing, as well as their informants were analyzed. The 10-item Functional Activities Questionnaire (FAQ), completed separately by patients (FAQ-S) and their informants (FAQ-I), assessed ADL function. Weighted $\kappa$ statistics summarized level of agreement, and a discrepancy score (FAQ-I - FAQ-S) quantified agreement. Correlation analyses between FAQ total scores, patient and informant characteristics, and cognitive scores were conducted, with post hoc regressions to determine the associations between both FAQ scores and cognition, independent of patient characteristics.

Results: The sample included 87 patients with normal cognition, 50 with mild cognitive impairment, and 13 with dementia. Overall, there was fair to moderate agreement between patients and informants on individual FAQ items $(0.27 \leq \kappa \leq 0.61, p<0.004)$, with greater discrepancies with increasing cognitive impairment. Patients' age, motor severity, non-motor burden, and depression also affected both ratings $(0.27 \leq r \leq 0.50$, $p<0.001$ ), with motor severity showing the greatest influence on both ratings. Both the FAQ-I and FAQ-S were correlated with almost all cognitive domains. Post hoc regression analyses controlling for patient characteristics showed that the attention domain was a significant predictor of both the FAQ-S and FAQ-I scores, and memory was also a significant predictor of the FAQ-I score. Only $29.3 \%$ of patients agreed perfectly with informants on the $F A Q$ total score, with informants most commonly rating $A D L$ impairments as more severe than patients. 


\begin{abstract}
Conclusions: Patient and informant ratings of $A D L$ function using FAQ items showed moderate agreement, with only few items reaching substantial agreement. Ratings of both were associated with patient cognitive status, but also other characteristics. In addition to patient and informant reports, objective measures are needed to accurately classify ADL deficits in PD.
\end{abstract}

Keywords: activities of daily living, caregiver, cognition, Functional Activities Questionnaire, Parkinson's Disease, self-ratings, informant-ratings

\section{INTRODUCTION}

Significant impairments in activities of daily living (ADL) function, in addition to impaired cognition, are the core criterion for diagnosing Parkinson's Disease (PD) dementia (PDD) (Emre et al., 2007). Both ADL impairments and severe cognitive impairment result in increased risk for nursing home placement and mortality (Hosking et al., 2021). Recent studies have shown that even patients with mild cognitive impairment (PD-MCI) display first signs of ADL dysfunction (Pirogovsky et al., 2014; Cheon et al., 2015; Fellows and Schmitter-Edgecombe, 2019), possibly indicating a group at risk for dementia (Beyle et al., 2018). As the diagnosis of ADL deficits requires these to be solely caused by cognitive deficits, an important step for accurate diagnoses is the measurement of ADL deficits in PD.

Insight into patients' general ADL function is commonly given by a reliable informant, such as a spouse or close friend (CahnWeiner et al., 2007). Research in Alzheimer's Disease shows that while informant reporting accurately reflects the functional changes, there are variations in the quality of their reports (Farias et al., 2017). Furthermore, caregiver stress and any depressive symptoms of the caregiver have an influence on the external assessment of ADL in mild dementia patients (Zanetti et al., 1999; Razani et al., 2007). Factors such as caregiver age, education level, living situation, and the nature of the relationship to the patient have been reported to influence the caregivers' assessments in Alzheimer's Disease (Lin et al., 2017), and also in PD (Bhimani, 2014; Mosley et al., 2017; Kalampokini et al., 2020). Additional negative influences on caregivers of $\mathrm{PD}$ patients include cognitive status, disease duration, and patients' motor symptom severity (Caap-Ahlgren and Dehlin, 2002; Ransmayr, 2020).

Self- reports are also used to gain perspective on how ADL impairments affect the patient's functioning (Foster and Hershey, 2011). It is important to note that the assessment of ADL impairments in elderly patients becomes more difficult with increasing cognitive deficits; patients with dementia often lack insight to correctly perceive the severity of their illness (Farias et al., 2017). PD patients also tend to underestimate their abilities with increasing cognitive decline (Seltzer et al., 2001), and previous studies have shown PD patients rate themselves as less impaired on measures of ADL than their caregivers (Leritz et al., 2004). Compared to objective measures, $44 \%$ of all study participants underestimated their ADL impairment, while 13\% overestimated impairment (Shulman et al., 2006). Patients who underestimated ADL disabilities had shorter disease durations, more preserved cognitive abilities, and were living in a family environment, while those who overestimated their ADL skills had advanced PD, showed cognitive dysfunctions, and lived alone (Shulman et al., 2006).

In contrast to the above-mentioned studies, other researchers have not been able to find differences in caregiver versus selfreport of ADL disabilities in PD patients (Brown et al., 1989; Liepelt-Scarfone et al., 2013; Copeland et al., 2016). More studies are needed to determine whether self-reports or informantreports are more useful for judging impairments in ADL function in the clinical routine, as the loss of functional independence is an important outcome of disease progression (Santos Garcia et al., 2021). The aim of this study was therefore to examine both self- and informant-reported ADL using a widely known questionnaire in a cohort of PD patients with varying degrees of cognitive impairment. We aimed to look at the agreement between both sources as well as associations with both patient and informant characteristics, hypothesizing that there would only be moderate agreement between both sources regarding $\mathrm{ADL}$ function, with increasing divergence relating to the severity of cognitive impairment.

\section{MATERIALS AND METHODS}

\section{Design and Recruitment}

Between July 2018 and September 2020, 270 PD patients were recruited to take part in the cross-sectional "Cognitivedriven ADL impairment as a predictor for Parkinson's disease dementia (PDD)" study. Inclusion criteria included: age between 50 and 90 years of age, diagnosis of PD according to UK Brain Bank Criteria, and the ability to understand study requirements and communicate with investigator. Exclusion criteria included: other neurodegenerative disease interfering with cognition or preventing the ability to give informed consent, alcohol, medication or drug dependency or abuse (except for nicotine), or participation in a clinical investigation of a new compound within the last 4 weeks. Additionally, all patients were asked to designate one person to be their informant who was then contacted to give information regarding the patient. This study was approved by the Ethics Committee of the Medical Faculty of the University of Tübingen (284/2018BO1). All patients (or their proxies if necessary) and their informants provided written, informed consent.

Of all patients invited to participate, 36 (13.3\%) met exclusion criteria and $52(19.3 \%)$ declined to take part in the examination. A total of $182(67.4 \%)$ patients were included in the study and assessed. For the following final analyses, 17 (9.3\%) patients who had received deep brain stimulation (DBS) implantation prior to 
assessment were excluded. We chose to exclude these patients as it is currently unclear how DBS affects ADL: some studies have shown improvements on ADL functions (Gorecka-Mazur et al., 2019; Cernera et al., 2020), while others demonstrated no effect on ADL function (Pusswald et al., 2019). Two patients (1.1\%) were additionally excluded as medication intake interfered with correct classification of cognitive status. Thirteen $(7.1 \%)$ patients had missing FAQ data ( $n=9$ did not have an informant and $n=4$ unable to fill out the FAQ themselves due to severely impaired cognition) and were also excluded. In total, data of 150 patients (and their informants) was analyzed in the final dataset.

\section{Assessments}

The Functional Activities Questionnaire (FAQ) (Pfeffer et al., 1982) was used to assess ADL impairments. It consists of 10 items assessing instrumental ADL functions, where capability of each item is rated from 0 (normal) to 3 (dependent on others). A maximum score of 30 points can be achieved, and higher scores indicate greater severity of ADL impairment. The FAQ was separately administered to both the patient as a self-report (FAQ-S) and the informant (FAQ-I) to evaluate the patients' ADL functioning in the previous 4 weeks.

\section{Patient Measures}

Patient demographics (age, sex, education years) and medical history [age at onset of PD, disease duration, and antiparkinsonian medication intake expressed using the levodopa equivalent daily dose (LEDD) (Tomlinson et al., 2010)] were collected. Motor function was assessed by a trained specialist using the Movement Disorder Society Unified Parkinson's Disease Rating Scale Part III (MDS-UPDRS-III) (Goetz et al., 2008). Depression was assessed using the Beck Depression Inventory-II (BDI-II) (Beck et al., 1996), a 21-item instrument quantifying depressive symptoms over the last 2 weeks. Non-motor symptom burden was assessed using the Parkinson's Disease Non-Motor Symptoms Questionnaire (NMSQ) (Chaudhuri et al., 2006).

Global cognitive functioning was measured using the Montreal Cognitive Assessment (MoCA) (Nasreddine et al., 2005). A comprehensive neuropsychological battery assessing five cognitive domains was administered:

- Attention: Letter-Number-Sequencing and Digit-Symbol subtests of the Wechsler Intelligenztest für Erwachsene [WIE, German adaptation of the Wechsler Intelligence Test for Adults, (Aster et al., 2006)];

- Executive Functions: Trail Making Test-Part B, semantic fluency, and phonemic fluency subtests from the Consortium to Establish a Registry for Alzheimer's Disease-Plus Battery (CERAD-PLUS) (Morris et al., 1988);

- Memory: Word List learning, Word List recall, Word List discriminability, and Constructional Praxis recall subtests of the CERAD-PLUS;

- Visuospatial Functions: Constructional Praxis subtest of the CERAD-PLUS and Fragmented Words subtest of the "Leistungsprüfsystem $50+$ " [performance test for older adults 50-90 years, (Sturm et al., 1993)];
- Language: Similarities subtest from the WIE and the modified Boston Naming Test of the CERAD-PLUS.

All raw scores were converted to $z$-scores using test manuals, adjusting for age and/or education where appropriate, and composite domain $z$-scores were calculated for each cognitive domain. Cognitive tests assigned to each domain were chosen according to the recommendations of the Movement Disorders Task Force (Litvan et al., 2012) and this comprehensive battery been previously used in a study with PD patients (Becker et al., 2020). The CERAD-PLUS battery has been shown to be accurate and useful in identifying cognitive impairment in PD patients (Karrasch et al., 2013; Camargo et al., 2018), while both the WIE [English version: Wechsler Adult Intelligence Scale (Yamawaki et al., 2020; Chen et al., 2021)] and the Leistungsprüfsystem $50+$ batteries have been utilized in PD-cognition studies (Fengler et al., 2016; Kalbe et al., 2016). Furthermore, a recent systematic literature review identified specific tests used in PD research that have been normed for German-speaking populations and their corresponding cognitive domain (Liepelt-Scarfone et al., 2021). The authors presented guidelines for the neuropsychological assessment of PD patients in the German language, with their findings supporting our chosen tests and domains.

Patients were classified as cognitively normal (PD-CN) if no cognitive or ADL impairment was present. PD-MCI was diagnosed according to the Level-II criteria of the Movement Disorders Task Force (Litvan et al., 2012) if impairment (1.5 standard deviations below population norms) was present in at least two cognitive tests, with preserved ADL functioning. PDD was diagnosed according to consensus criteria (Dubois et al., 2007 ) if both cognitive impairment and severe impairments in ADL function were present. Fourteen patients $(n=1 \mathrm{PD}-\mathrm{CN}$, $n=4 \mathrm{PD}-\mathrm{MCI}$, and $n=9 \mathrm{PDD})$ were unable to complete the neuropsychological test battery for various reasons (e.g., severe cognitive impairment, physical and/or mental exhaustion especially toward the end of the test battery). Cognitive diagnoses for these patients were made according to available cognitive data ( $z$-scores of the tests the patients did complete), agreements between informants and clinicians regarding ADL status, and neuropsychological investigator judgment. Available medical data (e.g., if the patient had received a diagnosis of either PD-MCI or PDD from a neuropsychologist, neurologist, or primary physician prior to examination) and previous cognitive evaluations were also taken into consideration.

\section{Informant Measures}

Demographic information was collected from each informant, including age, sex, education years, living situation, and how many times per week $(1 \times$ a week, $2-3 \times$ per week, or daily) they saw the patient. The Bayer-ADL scale (Hindmarch et al., 1998) was given to informants to assess instrumental ADL, where the patient's ability to perform 25 tasks is rated from 0 "never" to 10 "always." The total sum score is divided by the number of questions answered to obtain a scaled score, ranging from 1 to 10 , where higher values indicate more severe impairments in ADL function. Scoring is as follows: 1.0-2.0, no difficulties with ADL, 
TABLE 1 | Patient characteristics according to cognitive status.

\begin{tabular}{|c|c|c|c|c|}
\hline & PD-CN $n=87$ & PD-MCI $n=50$ & PDD $n=13$ & $p$-value \\
\hline \multicolumn{5}{|l|}{ Demographics } \\
\hline Male sex: $n(\%)$ & $58(66.7)$ & $27(54)$ & 9 (69.2) & 0.30 \\
\hline Age $(y)$ & 68.45 (51.99-83.47) & 68.13 (52.97-83.67) & 73.35 (67.69-82.30) & 0.17 \\
\hline Education (y) & $13(8-21)$ & $12(8-19)$ & $12(11-18)$ & $0.02^{*}$ \\
\hline Age at onset (y) & $60.48(39-75.54)$ & 58.77 (44.75-76.58) & $64.88(54.17-76.65)$ & 0.45 \\
\hline Disease duration (y) & 7.07 (3.28-21.74) & $8.05(2.15-20.22)$ & 7.42 (5.57-13.92) & 0.36 \\
\hline UPDRS-III & $22.5(3-50)$ & $30(8-68)$ & $45(27-56)$ & $<0.001^{* *}$ \\
\hline LEDD & 700 (100-1950) & 702 (52-1510) & $540(285-1050)$ & 0.45 \\
\hline NMSQ & $8(1-22)$ & $9.5(0-24)$ & $11(8-23)$ & $0.03^{*}$ \\
\hline BDI-II & $8(0-35)$ & $10(0-33)$ & $12(4-27)$ & $0.02^{*}$ \\
\hline \multicolumn{5}{|l|}{ ADL } \\
\hline FAQ-I total score & $1(0-24)$ & $3.50(0-24)$ & $19(7-28)$ & $<0.001^{\star *}$ \\
\hline FAQ-S total score & 0 (0-22) & $1(0-24)$ & $12(1-28)$ & $<0.001^{\star *}$ \\
\hline Bayer-ADL & $1.42(1-6.12)$ & $2.32(1-7.20)$ & 6.33 (2.20-9.56) & $<0.001^{\star *}$ \\
\hline Cognition $\neq_{\ddagger}$ & & & & $<0.001^{\star *}$ \\
\hline MoCA total score & $27(19-30)$ & $24.5(17-30)$ & $18(17-19)$ & $<0.001^{\star \star}$ \\
\hline Attention & $0.20(-1.20-1.90)$ & $-0.45(-2.60-1.20)$ & $-1.10(-2.10-0.70)$ & $<0.001^{\star *}$ \\
\hline Executive functions & $0.27(-2.00-2.57)$ & $-0.83(-1.93-1.27)$ & $-1.15(-1.83--0.70)$ & $<0.001^{\star *}$ \\
\hline Memory & $0.29(-1.90-1.30)$ & $-1.05(-2.95-0.38)$ & $-1.87(-2.90--0.77)$ & $<0.001^{\star \star}$ \\
\hline Visuospatial functions & $-0.15(-1.70-1.45)$ & $-1.20(-2.50-1.00)$ & $-1.62(-2.65--1.30)$ & $<0.001^{\star \star}$ \\
\hline Language & $0.43(-0.70-1.40)$ & $-0.30(-1.85-1.35)$ & $-0.47(-0.70--0.05)$ & $<0.001^{\star \star}$ \\
\hline
\end{tabular}

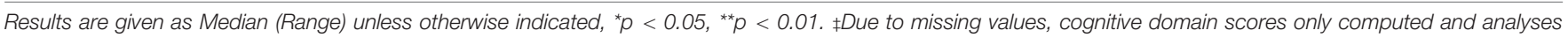

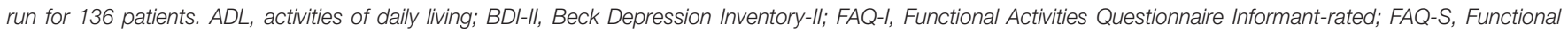

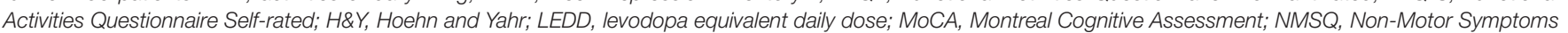

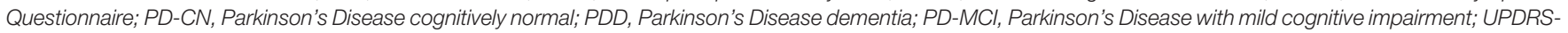
III, Unified Parkinson's Disease Rating Scale Part III; y, year.

2.1-5.0 mild difficulties with everyday function, and 5.1-10.0 indicates clear difficulties in coping with everyday life.

\section{Statistical Analyses}

REDCap electronic data capture tools (Harris et al., 2009) hosted at the Hertie Institute for Clinical Brain Research was used to collect and manage study data. SPSS Version 27 (IBM Corp., Armonk, NY, United States) was used to conduct all statistical analyses, with $\alpha$ levels set at 0.05 . For missing demographic patient data $[n=12(8 \%)$ MDS-UPDRS-III, $n=4(2.7 \%)$ NMSQ, and $n=2(1.3 \%)$ BDI-II], median values per cognitive group status were imputed to compensate for any missing values. Analyses involving cognitive data only included only those patients who had completed all tests $(n=136)$ to ensure equal representation of each averaged domain $z$-score. The ShapiroWilk tested assumptions of normality. As data were not normally distributed, demographic variables were examined using the non-parametric Pearson Chi-square, Mann-Whitney $U$ tests, or Independent-Samples Jonckheere-Terpstra Tests for Ordered Alternatives where appropriate.

Weighted $\kappa$ statistics with linear weights were used to summarize the level of agreement between patient and informants for each individual FAQ item. Agreement values were interpreted as follows: $0-0.20$ slight, $0.21-0.40$ fair, $0.41-0.60$ moderate, $0.61-0.80$ substantial, and $0.81-1.00$ almost perfect (Landis and Koch, 1977). The FAQ total score was compared between raters using Intraclass Correlation (ICC) using an absolute-agreement, two-way mixed effects model based on single measurements. To determine how different factors may influence the level of agreement, analyses were re-run using the following stratifications: (i) cognitive status of the patient [non-demented (PD-CN and PD-MCI) and demented (PDD)], (ii) sex of the patient, (iii) disease duration using a median split (duration $\leq 7.31$ years and duration $>7.32$ years), and (iv) BDI-II using a median split (score $\leq 9$ and score $>10$ ).

Spearman's rank correlations between the total FAQ-S and FAQ-I scores, cognitive scores, patient demographic variables of interest (age, sex, education, disease duration, UPDRSIII total score, BDI-II score, and NMSQ score), caregiver variables of interest (age, education years), and the Bayer-ADL were conducted. Post hoc multivariate linear regressions were conducted to determine the associations between both FAQ scores and cognition, independent of patient characteristics. Ten regressions were run for each of the five cognitive domains separately (due to multicollinearity when all domains are added into one model), with both the FAQ-S and the FAQ-I as the dependent variable. Further covariates in the models included patient age, UPDRS-III score, NMSQ scores and BDI-II score, as these were all significantly correlated with both FAQ scores. For these regressions, the $\alpha$ was adjusted to 0.005 to correct for multiple comparisons $(0.05 / 10)$.

Lastly, a discrepancy score (D) was calculated for the total FAQ score between informant and self-ratings: FAQ-I - FAQ-S. Positive values denoted higher impairment rated by informants 
( $\left.\mathrm{D}_{\mathrm{I}}\right)$, negative values denoted higher impairment rated by patients themselves $\left(D_{S}\right)$, and scores of 0 indicated perfect agreement between informants and patients $\left(\mathrm{D}_{\mathrm{A}}\right)$. Patients were split according to the discrepancy score, and demographic and cognitive variables were compared between groups using independent samples Kruskal-Wallis $H$ tests, with post hoc Bonferroni corrections for multiple testing.

\section{RESULTS}

Of all $150 \mathrm{PD}$ patients, 87 (58\%) were classified as PD-CN, 50 (33.3\%) as PD-MCI, and $13(8.7 \%)$ as PDD. The JonckheereTerpstra test showed a significant effect of education between groups, and post hoc analyses with Bonferroni correction for multiple tests revealed PD-MCI patients had significantly lower years of education than PD-CN patients $(p=0.03$; see Table 1 for details). Significant differences were found between all three groups for the UPDRS-III total score, where PDD patients had the most severe motor impairment according to post hoc analyses (PD-CN $<$ PD-MCI $<$ PDD, $p<0.002)$. Analyses also showed PDD patients also had a significantly higher non-motor symptom burden than PD-CN patients (post hoc $p=0.03$ ). The BDIII was statistically different between groups, however, post hoc significances did not reveal any specific group differences after correction for multiple testing. For measures of ADL, both the FAQ-I and FAQ-S total scores as well as the Bayer-ADL score were significantly different between groups, with PDD patients again showing the most severe impairments in ADL (post hoc $\mathrm{PD}-\mathrm{CN}<\mathrm{PD}-\mathrm{MCI}<\mathrm{PDD}, p<0.001)$.

Comparisons of cognitive data were done using data of 136 patients with complete neuropsychological testing (PD-CN $n=86$, PD-MCI $n=46$, PDD $n=4)$. Global cognition measured using the MoCA showed significant differences between groups, with PDD patients exhibiting the most impaired cognitive performance (post hoc PD-CN $>$ PD-MCI $>$ PDD, $p<0.008$ ). All five cognitive domains were significantly different between groups, following the same post hoc pattern where PD-MCI and PDD patients performed similarly, and PD-CN patients performed the best (PD-CN $>$ PD-MCI $=$ PDD, $p<0.005)$.

Regarding informants, they were most frequently spouses $(118,78.7 \%)$, followed by children/stepchildren (13, 8.7\%), life partner $(8,5.3 \%)$, close friend $(6,4 \%)$, other relative $(3,2 \%)$, and siblings $(2,1.3 \%)$ of the patients. Informants were also predominantly females $(101,67.3 \%)$, with a median age 64 (range 29-86) and median of 13 years total education (range 8-28). Regarding time spent with the patient, 121 (87.7\%) informants reported seeing them daily, $10(7.2 \%)$ two to three times a week, and 7 (5.1\%) only once a week.

\section{Agreement Statistics}

Table 2 shows the weighted $\kappa$ statistics for each FAQ item when examining the entire sample. Item 10 (traveling out of house) was rated as substantial agreement $(\kappa=0.61, p<0.01)$, with most other items reaching moderate or fair agreement $(0.27 \leq \kappa \leq 0.59, p<0.004)$. A good degree of reliability was found between patient and informant total scores on the FAQ. The single measure ICC was 0.73 with a $95 \%$ confidence interval $0.61-0.81[F(149,149)=7.24, p<0.001]$. Splitting patients according to cognitive status revealed that nondemented (PD-CN and PD-MCI) patients and their informants showed fair to moderate agreement on all items $(0.24 \leq \kappa \leq 0.57$, $p<0.004$ ), but only 3 items (1, 5, and 10) actually reached the moderate level (see Table 3). Agreement between demented patients and their informants was due to chance for almost all items $(0.08 \leq \kappa \leq 0.38, p>0.09)$. Only the FAQ items 3 (shopping alone) and 8 (paying attention) demonstrated moderate agreement $(\kappa=0.56$ and $\kappa=0.51$, respectively; $p<0.01)$ between patient and informant ratings.

Next, the role of sex of the patient was examined. There was a slight tendency for better agreements when the patient was a male than if they were females (Supplementary Table 1). The agreement of ratings for male patients ranged from fair to substantial $(0.30 \leq \kappa \leq 0.68, p<0.002)$, while ratings for female patients were fair to moderate $(0.23 \leq \kappa \leq 0.55, p<0.008)$ and one item with a slight agreement due to chance $(\kappa=0.10$, $p=0.30)$. Patients were then split according to median years of disease duration (Supplementary Table 2). For patients with a shorter disease duration, item 5 (using appliances) was rated as substantial $(\kappa=0.62, p<0.001)$, with all other items reaching a fair or moderate agreement $(0.22 \leq \kappa \leq 0.53, p<0.01)$. The group of patients with longer disease duration also showed fair to moderate agreement $(0.24 \leq \kappa \leq 0.46, p<0.01)$, and items 1 (handling finances) and 10 (traveling out of house) reached substantial agreement $(\kappa=0.62$ and $\kappa=0.69$, respectively; $p<0.001)$. Lastly, patients were split according to median BDIII score (Supplementary Table 3). Those patients with lower depressive symptomatology demonstrated substantial agreement on three items $(0.62 \leq \kappa \leq 0.72, p<0.001)$, while the others showed fair or moderate agreement $(0.31 \leq \kappa \leq 0.52, p<0.001)$. Patients with higher depressive symptoms showed overall less agreement than those with higher symptoms. Almost all items had fair to moderate agreement $(0.32 \leq \kappa \leq 0.59, p<0.01)$, while item 4 (skills and hobbies) had only slight agreement $(\kappa=0.17$, $p=0.02)$.

For all agreement analyses run, the number of ratings of items $\geq 1$ (indicating at least mild difficulties with the daily task) was consistently higher for informant ratings than for the self-ratings. We examined the consistency of the individual item agreement statistics post hoc, to determine whether some FAQ items consistently showed better or worse agreement than others. This was done by ranking the weighted $\kappa$ statistics of all analyses according to FAQ item and examining the frequency of the items corresponding to the top and bottom three ranks. There was a clear tendency for the items 10 (traveling out of house), 1 (handling finances and balancing checkbook), and a tie between 3 (shopping alone) and 5 (using household appliances) to show the most agreement (top ranked in $8,7,4$, and 4 analyses, respectively). The items 4 (engaging in skills and hobbies), 6 (preparing a balanced meal), and 7 (keeping up with current events) were those with the poorest consistent agreement (bottom ranked in 8, 6, and 6 analyses, respectively).

\section{Correlation Analyses}

The FAQ-S and FAQ-I total scores were moderately positively correlated with one another $\left(r_{s}=0.61, p<0.001\right)$. Table 4 shows 
TABLE 2 | Differences between self and informant ratings of the FAQ.

\begin{tabular}{|c|c|c|c|c|c|}
\hline FAQ item & Self & Informant & Weighted $\kappa$ & SE of $\kappa$ & $p$-value \\
\hline 1. Handling finances & $20(13.3)$ & $36(24)$ & 0.59 & 0.08 & $<0.001^{* \star}$ \\
\hline 2. Assembling tax records & $47(31.3)$ & $56(37.3)$ & 0.47 & 0.07 & $<0.001^{\star *}$ \\
\hline 3. Shopping & $27(18)$ & $41(27.3)$ & 0.48 & 0.08 & $<0.001^{\star \star}$ \\
\hline 4. Skills and hobbies & $21(14)$ & $58(38.7)$ & 0.27 & 0.06 & $<0.001^{\star \star}$ \\
\hline 5. Using appliances & $12(8)$ & $24(16)$ & 0.48 & 0.11 & $<0.001^{\star \star}$ \\
\hline 6. Meal preparation & $31(20.7)$ & $52(34.7)$ & 0.37 & 0.08 & $<0.001^{\star \star}$ \\
\hline 7. Current events & $13(8.7)$ & $26(17.3)$ & 0.37 & 0.10 & $<0.001^{\star *}$ \\
\hline 8. Paying attention & $20(13.3)$ & $30(20)$ & 0.40 & 0.08 & $<0.001^{\star *}$ \\
\hline 9. Remembering appointments & $37(24.7)$ & $48(32)$ & 0.39 & 0.07 & $<0.001^{* \star}$ \\
\hline 10. Traveling out of house & $26(17.3)$ & $42(28)$ & 0.61 & 0.07 & $<0.001^{* *}$ \\
\hline
\end{tabular}

Results are expressed as Number of patients scoring $\geq 1$ (\%), ${ }^{* *} p<0.01$. FAQ, Functional Activities Questionnaire; SE, standard error.

TABLE 3 | Agreement between self and informant ratings of the FAQ according to cognitive status.

\begin{tabular}{|c|c|c|c|c|c|c|c|c|c|c|}
\hline \multirow[b]{2}{*}{ FAQ item } & \multicolumn{5}{|c|}{ Non-demented PD patients $n=137$} & \multicolumn{5}{|c|}{ Demented PD patients $n=13$} \\
\hline & Self & Informant & Weighted $\mathrm{\kappa}$ & SE of $\kappa$ & $p$-value & Self & Informant & Weighted $\kappa$ & SE of $k$ & $p$-value \\
\hline 1. Handling finances & $11(8)$ & $23(16.8)$ & 0.51 & 0.11 & $<0.001^{\star *}$ & $9(69.2)$ & $13(100)$ & 0.17 & 0.12 & 0.18 \\
\hline 2. Assembling tax records & $37(27)$ & $43(31.4)$ & 0.37 & 0.08 & $<0.001^{\star \star}$ & $10(76.9)$ & $13(100)$ & 0.08 & 0.07 & 0.26 \\
\hline 3. Shopping & $19(13.9)$ & $30(21.9)$ & 0.38 & 0.09 & $<0.001^{\star *}$ & $8(61.5)$ & $11(84.6)$ & 0.56 & 0.17 & $0.002^{\star *}$ \\
\hline 4. Skills and hobbies & $14(10.2)$ & $50(36.5)$ & 0.24 & 0.07 & $<0.001^{\star *}$ & $7(53.8)$ & $8(61.5)$ & 0.13 & 0.18 & 0.52 \\
\hline 5. Using appliances & $7(5.1)$ & $15(10.9)$ & 0.45 & 0.15 & $<0.001^{\star *}$ & $5(38.5)$ & $9(69.2)$ & 0.29 & 0.20 & 0.15 \\
\hline 6. Meal preparation & $24(17.5)$ & $41(29.9)$ & 0.29 & 0.08 & $<0.001^{\star \star}$ & 7 (53.8) & $11(84.6)$ & 0.21 & 0.19 & 0.28 \\
\hline 7. Current events & $10(7.3)$ & $18(13.1)$ & 0.39 & 0.11 & $<0.001^{\star *}$ & $3(23.1)$ & $8(61.5)$ & 0.18 & 0.22 & 0.33 \\
\hline 8. Paying attention & 15 (10.9) & $24(17.5)$ & 0.33 & 0.09 & $<0.001^{\star *}$ & $5(38.5)$ & $6(46.2)$ & 0.51 & 0.14 & $0.01^{*}$ \\
\hline 9. Remembering appointments & $26(19)$ & $37(27)$ & 0.30 & 0.09 & $<0.001^{\star *}$ & $11(84.6)$ & $11(84.6)$ & 0.20 & 0.20 & 0.27 \\
\hline 10. Traveling out of house & 19 (13.9) & $32(23.4)$ & 0.57 & 0.09 & $<0.001^{\star *}$ & $7(53.8)$ & $10(76.9)$ & 0.38 & 0.19 & 0.09 \\
\hline
\end{tabular}

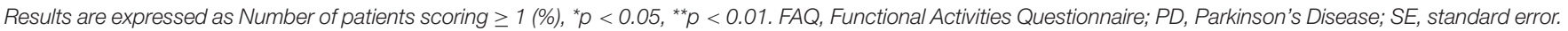

the correlations for the FAQ scores and both patient and caregiver variables of interest. Both the FAQ-S and FAQ-I total scores were positively correlated with patients' age, motor severity, number of non-motor symptoms, and depressive symptoms $\left(0.27 \leq r_{s} \leq 0.50, p<0.001\right)$, and negatively correlated with patients' education $\left(r_{s}=-0.21\right.$ and $r_{s}=-0.18$, respectively; $p<0.03)$. The Bayer-ADL score was strongly positively correlated with the FAQ-I $\left(r_{s}=0.85, p<0.001\right)$ and moderately correlated with the FAQ-S $\left(r_{s}=0.55, p<0.001\right)$. Examining the cognitive data, both the FAQ-S and FAQ-I scores were negatively associated with the MoCA $\left(r_{s}=-0.33\right.$ and $r_{s}=-0.38$, respectively; $p<0.001$ ). The FAQ-S was significantly negatively correlated with the attention, executive functions, memory, and visuospatial functions domains $\left(-0.40 \leq r_{s} \leq-0.19, p<0.02\right)$, but not with language. In contrast, for the FAQ-I, there was a significant negative correlation with all cognitive domains $\left(-0.43 \leq r_{s} \leq-0.21, p<0.02\right)$.

\section{Post hoc Regression Analyses}

All ten linear regression models with either the FAQ-S or FAQI as the dependent variable were stable. The linear regression with attention as the cognitive independent and FAQ-S as the dependent variable was statistically significant $[F(5,130)=8.27$, $p<0.001$ ], explaining $24.1 \%$ (Nagelkerke $R^{2}$ ) of the variance. Attention significantly predicted the FAQ-S (unstandardized $\beta=-1.49$, standard error of $\beta=0.46, p=0.001$ ), as did the UPDRS-III score (unstandardized $\beta=0.09$, standard error of $\beta=0.03, p=0.004)$. The model including memory was also significant $[F(5,130)=7.50, p<0.001]$, explaining $22.4 \%$ (Nagelkerke $R^{2}$ ) of the variance. Memory domain as a predictor did not reach clinical significance after correction for multiple testing (unstandardized $\beta=-0.99$, standard error of $\beta=0.36$, $p=0.007$ ), while the UPDRS-III score was again a significant predictor (unstandardized $\beta=0.11$, standard error of $\beta=0.04$, $p=0.001$ ). For the FAQ-S models including executive functions, visuospatial functions, and language domains, only the UPDRSIII score was a significant predictor $(p<0.004)$.

When the dependent variable was the FAQ-I, the model including attention was again significant $[F(5,130)=10.48$, $p<0.001$ ], explaining $28.7 \%$ (Nagelkerke $R^{2}$ ) of the variance. Attention significantly predicted the FAQ-I (unstandardized $\beta=-1.75$, standard error of $\beta=0.56, p=0.002$ ), as did the UPDRS-III score (unstandardized $\beta=0.11$, standard error of $\beta=0.04, p=0.005$ ) and patient age (unstandardized $\beta=0.18$, standard error of $\beta=0.05, p<0.001$ ). When memory was the independent variable, the model was again significant $[F(5,130)=10.12, p<0.001]$, explaining 28\% (Nagelkerke $R^{2}$ ) of the variance. Significant predictors of the FAQ-I were memory (unstandardized $\beta=-1.27$, standard error of $\beta=0.44$, $p=0.004$ ), UPDRS-III score (unstandardized $\beta=0.12$, standard 
TABLE 4 | Correlations between the total FAQ-S and FAQ-I scores and both patient and caregiver variables of interest.

\begin{tabular}{|c|c|c|}
\hline & FAQ-S & FAQ-I \\
\hline \multicolumn{3}{|c|}{ Patient characteristics } \\
\hline Patient age & $0.27^{\star \star}$ & $0.34^{\star *}$ \\
\hline Patient education & $-0.21^{\star \star}$ & $-0.18^{\star}$ \\
\hline Disease duration & 0.05 & 0.13 \\
\hline UPDRS-III & $0.38^{\star \star}$ & $0.50^{\star *}$ \\
\hline NMSQ & $0.35^{\star \star}$ & $0.36^{\star \star}$ \\
\hline BDI-II & $0.27^{\star \star}$ & $0.35^{\star \star}$ \\
\hline \multicolumn{3}{|l|}{ Patient cognition $\neq$} \\
\hline MoCA total score & $-0.33^{\star \star}$ & $-0.38^{\star \star}$ \\
\hline Attention & $-0.38^{\star \star}$ & $-0.43^{\star \star}$ \\
\hline Executive functions & $-0.31^{\star \star}$ & $-0.36^{\star \star}$ \\
\hline Memory & $-0.40^{\star *}$ & $-0.31^{\text {** }}$ \\
\hline Visuospatial functions & $-0.19^{\star}$ & $-0.21^{\star}$ \\
\hline Language & -0.14 & $-0.21^{\star}$ \\
\hline \multicolumn{3}{|c|}{ Caregiver characteristics } \\
\hline Caregiver age & 0.06 & 0.11 \\
\hline Caregiver education & $-0.18^{\star}$ & -0.14 \\
\hline Bayer-ADL & $0.55^{\star \star}$ & $0.85^{\star \star}$ \\
\hline
\end{tabular}

${ }^{*} p<0.05,{ }^{* *} p<0.01$. $\neq$ Due to missing values, cognitive domain scores only computed and analyses run for 136 patients. ADL, activities of daily living; BDI-II, Beck Depression Inventory-II; FAQ-I, Functional Activities Questionnaire Informantrated; FAQ-S, Functional Activities Questionnaire Self-rated; MoCA, Montreal Cognitive Assessment; NMSQ, Non-Motor Symptoms Questionnaire; UPDRS-III, Unified Parkinson's Disease Rating Scale Part III.

error of $\beta=0.04, p=0.002$ ), and patient age (unstandardized $\beta=0.19$, standard error of $\beta=0.05, p<0.001$ ). The FAQ-S models including executive functions, visuospatial functions, and language domains, were significant, however, the only significant predictors were patient age $(p<0.001)$ and UPDRS-III score $(p<0.001)$.

\section{Discrepancy Scores}

The discrepancy score based on difference between the FAQ-S and FAQ-I total scores showed that 76 (50.7\%) of informants rated $A D L$ function as more impaired than their patients $\left(D_{I}\right.$ group), 44 (29.3\%) of participants agreed with their informants ( $\mathrm{D}_{\mathrm{A}}$ group), and 30 (20\%) patients rated their impairment worse than informants ( $D_{S}$ group). Cognitive group distribution was as follows: $\mathrm{D}_{\mathrm{I}}-38$ PD-CN, 27 PD-MCI, 11 PDD; $\mathrm{D}_{\mathrm{A}}-34$ PD-CN, 10 PD-MCI; $\mathrm{D}_{\mathrm{S}}-15$ PD-CN, 13 PD-MCI, 2 PDD.

Kruskal-Wallis $H$ tests showed group differences for patient age $[H(2)=6.59, p=0.04]$, and post hoc Bonferroni corrected analyses revealed patients in the $\mathrm{D}_{\text {I }}$ group were significantly older than patients in the $\mathrm{D}_{\mathrm{A}}$ group $(p=0.04)$. A significant effect was found for the MDS-UPDRS-III score $[H(2)=19.29, p<0.001]$, where motor severity was greater in the $\mathrm{D}_{\text {I }}$ group than in the $\mathrm{D}_{\mathrm{A}}$ group $(p<0.001)$. The NMSQ was also significantly different between groups $[H(2)=11.94, p=0.003]$, with patients in the $\mathrm{D}_{\mathrm{A}}$ group reporting less non-motor symptoms than both the $\mathrm{D}_{\mathrm{S}}$ $(p=0.03)$ and $\mathrm{D}_{\mathrm{I}}(p=0.003)$ groups. Depressive symptomatology was also different between groups $[H(2)=11.29, p=0.004]$, where patients in the $\mathrm{D}_{\mathrm{I}}$ group had higher BDI scores than those in the
$\mathrm{D}_{\mathrm{A}}$ group. The Bayer ADL scale score was significantly different between groups, $[H(2)=57.82, p<0.001]$, with post hoc analyses showing $\mathrm{D}_{\mathrm{A}}<\mathrm{D}_{\mathrm{S}}<\mathrm{D}_{\mathrm{I}}, p<0.02$ for all. Neither caregiver age $[H(2)=0.66, p=0.72]$ nor education level $[H(2)=2.15, p=0.34]$ were significantly different between groups.

For the cognitive data, a significant effect for the MoCA was found $[H(2)=9.47, p=0.009]$, where patients in the DI group had significantly lowered global cognition than patients in the DA group $(p=0.006)$. The attention domain showed a significant group effect $[H(2)=22.49, p<0.001]$ where patients in the $\mathrm{D}_{\mathrm{A}}$ had significantly better cognition than patients in both the $D_{S}$ and $\mathrm{D}_{\text {I }}$ groups $(p<0.01)$. Significant group effects were also found for the executive functions $[H(2)=13.45, p=0.001]$, memory $[H(2)=9.85, p=0.007]$, and visuospatial functions $[H(2)=9.68$, $p=0.008$ ] domains. Post hoc results showed patients in the $\mathrm{D}_{\mathrm{A}}$ had significantly better cognition than patients in the $\mathrm{D}_{\mathrm{I}}$ groups $(p<0.01)$. For the visuospatial domain, patients in the $\mathrm{D}_{\mathrm{A}}$ had borderline insignificant $(p=0.05)$ better cognitive scores than patients in the $D_{S}$ group. No effect was found between groups for performance in the language domain $[H(2)=1.62, p=0.45]$. Figure 1 shows a box-and-whisker plot of performance on each cognitive domain across discrepancy score groups including post hoc significant differences.

\section{DISCUSSION}

This study aimed to examine the agreement between self- and informant-reports of ADL function and determine whether they were affected by different patient or informant characteristics. The main results were that: (i) agreement analyses showed overall fair to moderate agreement, with few items reaching substantial agreement in various sub-analyses; (ii) both patient and informant ratings were significantly correlated with patient characteristics including cognition, as well as motor severity, age, and depression; and (iii) informants most commonly rated ADL impairments as more severe than patients, with only $29.3 \%$ of patients showing perfect agreement with informants on the their ADL performance as reflected by the FAQ total score.

Severe deficits in daily functioning are the core criteria for diagnosing PDD, however, studies have shown that even PD-MCI patients can show early, non-clinical signs of ADL dysfunction (Pirogovsky et al., 2014; Beyle et al., 2018). It is important to understand how and when changes in $\mathrm{ADL}$ indicative of dementia in PD emerge to be able to provide early interventions for maintaining patients' autonomy and, as a direct consequence, their quality of life. However, to be able to recognize significant changes in ADL indicating first signs of PDD, reliable and valid assessments with high diagnostic accuracy are needed. Our current results showed that when using the FAQ, there was only a fair to moderate agreement between patients and their informants regarding the daily functional abilities of the patient. The data also support previous findings that there was some level of over- and underreporting done by either patients or caregivers (Shulman et al., 2006; Christ et al., 2013; Cholerton et al., 2020), which is important to take into account when using these questionnaires to screen for $\mathrm{ADL}$ impairment indicative 


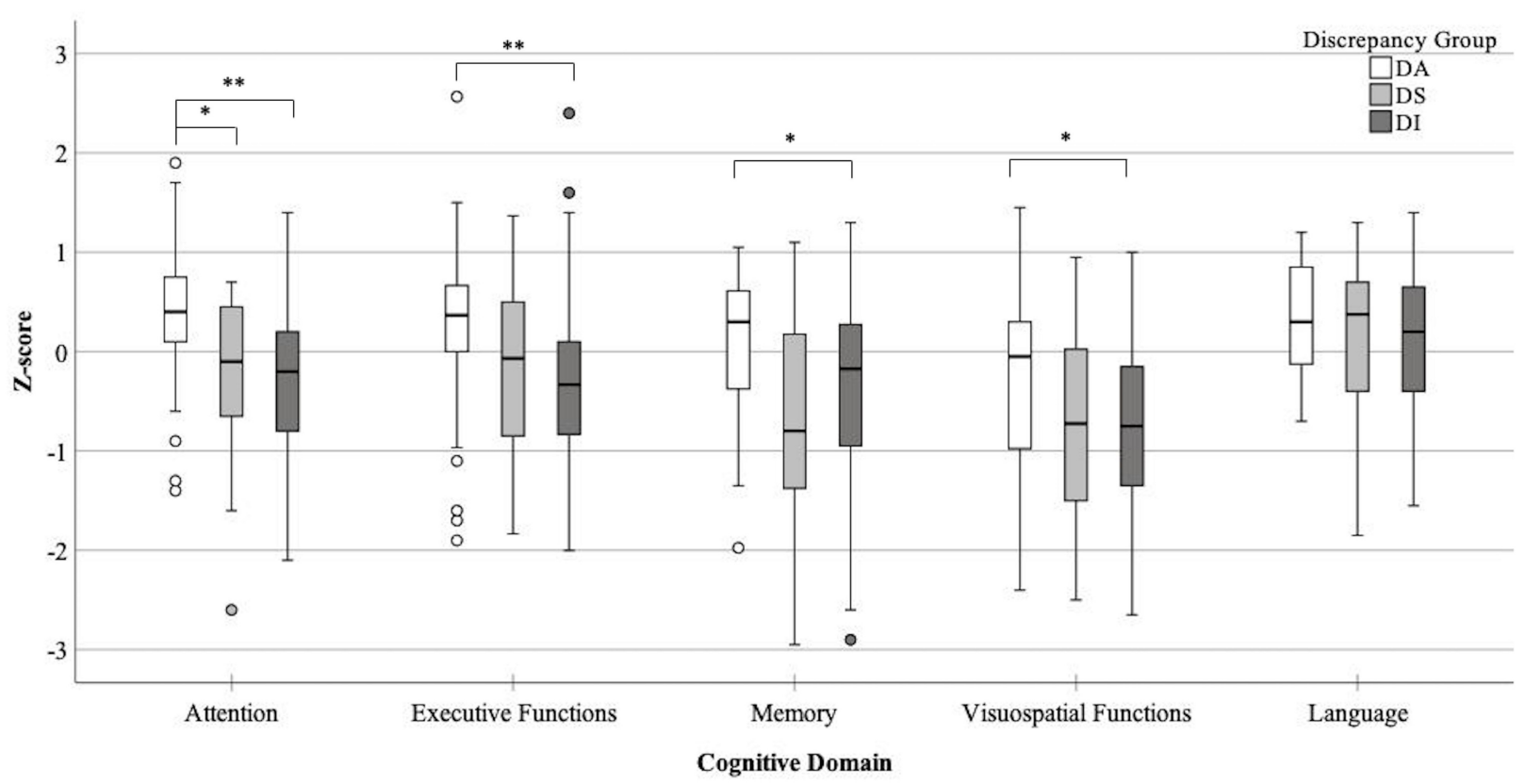

FIGURE 1 | Box-and-whisker plot detailing performance across all five cognitive domains for each discrepancy score group (DA- perfect agreement between informants and patients, DS- higher impairment rated by patients, DI- higher impairment rated by informants), ${ }^{*} p<0.05, * * p<0.001$.

of dementia. To the authors knowledge, only one other study has directly examined inter-rater agreement of ADL ratings by giving both patients and informants the same scale (Deck et al., 2019). Patient and caregiver agreement was examined with the Penn Parkinson's Daily Activities Questionnaire (PDAQ-15), a scale designed to assess instrumental cognitive-associated ADL impairment in PD, and additionally compared to an objective functional measure of ADL. The authors of this study found a moderate agreement between patient and informant ratings on the total (ICC $=0.57$ ) and individual items of the PDAQ-15 (Deck et al., 2019), similar to our current findings reported for the FAQ. Together these results show that agreement between patients and informants is not perfect, regardless of the scale used and the underlying constructs measured. It is imperative to for clinicians and researchers alike to consider that using a single measure of ADL dysfunction, whether rated by the patient or informant, to detect and diagnose presence of PDD potentially overestimates the motor effects on ADL function as well as underestimating cognitive sources. Incorporating objective measures of functional performance as well as self or informant-reported questionnaires may aid in determining level of ADL functioning especially in PD-MCI patients who would be able to undergo rigorous testing. Alternatively, consensus rating between both patient and informants should be considered, although studies are needed to determine whether these will better correspond to patient's real-world abilities than individual assessments.

A specific item analysis was undertaken which showed that traveling out of house, handling finances, going shopping alone, and using household appliances had the highest agreement in our sample, while engaging in skills and hobbies, preparing a balanced meal, and keeping up with current events had the poorest agreement. A previous study showed the specific ADL items managing finances, keeping their appointments, following current events, and using a phone were unaffected by motor symptoms and able to identify dementia in PD patients (Cheon et al., 2015). Furthermore, differentiating cognitive from motor influences on the FAQ showed that both handling finances and keeping up with current events were predicted by cognitive, not motor, abilities (Becker et al., 2020). However, only finances showed adequate agreement within our sample, whereas the agreements between patients and informants regarding following current events were consistently poor. It is possible that impairments in handling finances can be better acknowledged by patients because there is a tangible result which may be cause for concern when impairments are noted, while keeping up with current events is a more subjective experience. Patients may tend to overestimate this ability as they lack a concrete way to measure its loss. On the other hand, traveling out of the house, going shopping alone, and using appliances were all related to motor ADL - as these are affected by PD motor symptoms (e.g., tremor or dyskinesias), it is possible that these are more often noticed by patients and informants alike. This is also supported by the fact that motor severity was a significant factor in predicting both FAQ-S and FAQ-I scores. Patients may be more willing to admit their difficulties in these areas as opposed to, for example, engaging in their skills and hobbies which they are more hesitant to give up due to motor symptoms. Future studies should examine in further detail whether there are specific ADL abilities that may be more prone to disagreements and how both patient and informant perceptions of these abilities can change over time. It is possible that either patient or informant ratings on certain $\mathrm{ADL}$ questionnaire items correspond accurately 
to patients' real-life functioning. Such analyses can inform judgments regarding severity of ADL deficits as more weight can be given to items and ratings known to reflect patients' daily life.

Generally, studies have focused on comparing informantrated and patient-rated $\mathrm{ADL}$ functioning to the objective cognitive performance of the patient (Shulman et al., 2006; Copeland et al., 2016; Cholerton et al., 2020). While both patients and their informants may not accurately identify specific cognitive deficits, these studies generally show that in early stages of cognitive decline, patient-ratings may be more sensitive to changes in ADL affected by cognition than those of their informants. Only with increasing cognitive decline does the participant lose awareness of their ADL abilities and the knowledgeable informant report become more valuable. Current results confirmed this previous research that PD patients underestimate their abilities with increasing cognitive decline by rating themselves as less impaired than their informants (Seltzer et al., 2001; Leritz et al., 2004; Shulman et al., 2006; Deck et al., 2019). There was poor agreement which was due to chance for almost all individual FAQ items in PDD patients, indicating that demented patients and their informants cannot agree on the patients' ability to carry out ADL. Due to the small sample size in our study, however, data needed to be interpreted with caution and validated in larger samples. When examining non-demented patients, agreement ratings tended to be worse than when examining the entire sample. This is interesting, as anosognosia is not necessarily found in earlier stages of cognitive decline, although reports are varied (Pennington et al., 2021). We also examined associations directly with cognitive domains. Both patient and informant ratings were correlated with almost all cognitive domains, showing that $\mathrm{ADL}$ impairments present in $\mathrm{PD}$ are again associated with cognitive decline. To determine whether and to what extent this effect was influenced by patient characteristics, we conducted additional regression analyses. These showed that both patient and informant FAQ ratings were predicted by patients' performance on attention and memory domains. Previously, it has been shown that deficits in attention predicted $\mathrm{ADL}$ performance in $\mathrm{PD}$, after controlling for confounders including age, sex, and motor impairment (Bronnick et al., 2006; Becker et al., 2020). Our results that attentional deficits, independent of motor or non-motor symptoms, increased ratings of ADL impairment corroborate previous results and highlight that attentional deficits may decline in parallel with ADL deficits. Studies have also demonstrated relationships between memory performance and ADL impairments (Beyle et al., 2018; Foster and Doty, 2021). Future research should confirm how memory and attention deficits impair patients' ADL function, which may lead to more person-centered interventions and assessment strategies.

Apart from cognition, both patient and informant ratings were influenced by patient characteristics, evidenced by both correlation and agreement analyses performed. Severity of PD symptoms, presence of depression, and disease-related motor complications in quality of life are associated with self-assessment of ADL function (Hobson et al., 2001). Our findings replicate most of these findings and also demonstrate they are associated with informant assessments, by showing that both the FAQ-I and FAQ-S were correlated with patients' age, motor severity, non-motor symptom burden, and depression symptoms. This is important as more than $90 \%$ of PD patients present with at least one NMS (Barone et al., 2009) and around 35\% present with clinically significant depressive symptoms (Reijnders et al., 2008). Clinicians evaluating ADL deficits should ask whether and to what extent these symptoms are affecting daily functioning, to ascertain a more reliable index of ADL function. Perhaps the most important finding is that patients' motor severity influences all ADL ratings, and even modulates the association between cognition and ADL ratings. Previous studies have shown that motor symptoms of PD affect ADL (Martinez-Martin et al., 2003; Skinner et al., 2015) and are independent of the cognitivedriven ADL aspects (Becker et al., 2020). However, the diagnosis of PDD requires ADL deficits to be caused by cognitive and not motor problems; as both patient and informant reports are influenced by motor severity, this further emphasizes the need for accurate ADL scales that can capture deficits independent of motor influences. Furthermore, we found an effect of the patients' sex, where there was a slight tendency for better agreement for male than female patients. This is in line with a previous study that found that women with PD were likely to report more severe ADL dysfunction (Medijainen et al., 2015). This is an interesting finding that should be replicated in future studies, as it would be crucial for clinicians to consider sex differences when judging ADL deficits. Notably, neither informant nor self-ratings were influenced by caregiver age or education, which is not in line with previous literature (Bhimani, 2014; Kalampokini et al., 2020). However, we did not evaluate more specific informant details, such as caregiver burden, depression, or social life, all of which have been shown to have an effect on the reporting of ADL function. Future studies are needed to determine more specifically how and to what extent these caregiver attributes affect their ratings, and whether there are ways to partialize these effects out.

Lastly, discrepancy scores were used to quantify the agreement between patients and informants. Generally, informants rated the ADL impairments as more severe than the patients did, and only $30 \%$ of patients and informants agreed regarding the total score. Notably, there were no PDD patients where there was an agreement between both raters, further confirming that PD patients show worse insight into their ADL deficits with worsening cognition. We found that the group where informants rated ADL deficits as more severe were older, had worse motor severity, higher non-motor burden, more depressive symptoms, and worse cognition (in the attention, executive function, memory, and visuospatial domains) than the group where patients and informants agreed. A previous study found patients who underestimated ADL disabilities had shorter disease durations, more preserved cognitive abilities, and were living in a family environment, while those who overestimated their ADL skills had advanced PD, showed cognitive dysfunctions, and lived alone (Shulman et al., 2006). It would be interesting for future studies to determine to what extent difficulties in ADL are results of these factors and the disease progression. Furthermore, studies should explore different factors related to either the patient (such as comorbid 
diseases, neuropsychiatric disturbances, or other common nonmotor symptoms including fatigue/sleep disturbances, or urinary dysfunction) or the informant (personality traits, quality of life, stress, and psychosocial burden) that could influence ADL function, and how these may affect ratings.

There are limitations of this study that need to be addressed. Most importantly, no objective measures of ADL function, such as performance-based tests which were used in Deck et al. (2019), were used to validate the ADL ratings of the FAQ in this study. Therefore, we cannot determine whether patients' or their informants' ratings correspond most accurately to the reallife functional performance. While performance-based tests have been used in PD research, these measures are often not feasible in clinical and research settings as they are time-consuming and can be heavily influenced by motor severity (Sulzer et al., 2020). Future studies should incorporate objective measures of ADL function to determine the relation of both patient and informant ratings to objective observed ADL performance and to overcome any methodological biases or inaccuracy errors that may be related to the use of questionnaires (Sadek et al., 2011). Moreover, the current analyses should be repeated using different ADL scales, to determine whether they show better or worse agreement between patients and informants, to aid both clinicians and researchers in their selection of assessment tools. The current results should also be interpreted with caution due to the imbalanced group sizes for each cognitive status (especially for the PDD group). Studies should seek to replicate current findings using not only equal sample sizes, but also using different neuropsychological tests or different cut-offs for diagnosis of cognitive impairment. Furthermore, as briefly mentioned, we did not evaluate more specific caregiver details, as the primary outcome of the study was changes in ADL functioning of patients (with information from informants supporting the evaluation of ADL function). Certain sub-analyses of caregiver variables also could not be performed in this study. Examination of whether relation to and time spent with the patient affected agreement were not possible due to a too-small group sizes for adequately powered analyses, and agreement statistics between different categories of caregiver relationship were unable to be run in certain cases due to variables being constant. More studies are needed to determine the extent that informant factors may have on their perceptions of patient ADL functions.

\section{CONCLUSION}

This study examined self- and informant-reported ADL using a widely known questionnaire in a cohort of $\mathrm{PD}$ patients with varying degrees of cognitive impairment by looking at the agreement between both raters as well as associations with both patient and informant characteristics. Results of the study showed that when using the same ADL questionnaire, there was only a fair to moderate agreement between patients and their informants regarding the patients' daily functioning, with divergence between ratings increasing as cognitive impairment becomes more severe. Overall, less than one-third $(29.3 \%)$ of PD patients had perfect agreement with informants on the
FAQ total score, highlighting again that this discrepancy is very pronounced. Our results also highlight that motor severity influences ADL ratings and modulates associations to cognition, Lastly, as the diagnosis of PDD necessitates impairments in $\mathrm{ADL}$ to be cognitive based, it is important for clinicians to understand that both informants and patients are affected by patient characteristics (most notably motor severity) and must take these into account and rule out their influences when deciding on a dementia diagnosis.

\section{DATA AVAILABILITY STATEMENT}

The datasets presented in this article are not readily available because the corresponding author will consider any requests for access to the data (including de-identified participant data and corresponding data dictionary) reported in this manuscript. Requests to access the datasets should be directed to IL-S, inga.liepelt@uni-tuebingen.de.

\section{ETHICS STATEMENT}

The studies involving human participants were reviewed and approved by the Ethics Committee of the Medical Faculty of University of Tübingen. The patients/participants provided their written informed consent to participate in this study.

\section{AUTHOR CONTRIBUTIONS}

SB, BF, and IL-S: design and conceptualization of study. SB, SS, $\mathrm{KM}$, and KB: data collection. SB and IL-S: statistical analysis. SB: writing of the first draft. SS, KM, BF, KB, and IL-S: review and critique of the manuscript. All authors contributed to the article and approved the submitted version.

\section{FUNDING}

This work was supported by a grant from the Michael J. Fox Foundation (MJFF) for Parkinson's Research (Grant Number 15227). Michael J. Fox Foundation was not involved in the study design, data collection, data analysis, decision to publish, or preparation of the manuscript. We also acknowledge support by the Open Access Publishing Fund of the University of Tübingen.

\section{ACKNOWLEDGMENTS}

We would like to thank all patients and informants who participated in this study.

\section{SUPPLEMENTARY MATERIAL}

The Supplementary Material for this article can be found online at: https://www.frontiersin.org/articles/10.3389/fnagi.2022. 838674/full\#supplementary-material 


\section{REFERENCES}

Aster, M., Neubauer, A., and Horn, R. (2006). WIE. Wechsler Intelligenztest Fuer Erwachsene. Deutschsprachige Bearbeitung und Adaptation des WAIS-III von David Wechsler. Bern: Hogrefe.

Barone, P., Antonini, A., Colosimo, C., Marconi, R., Morgante, L., Avarello, T. P., et al. (2009). The PRIAMO study: a multicenter assessment of nonmotor symptoms and their impact on quality of life in Parkinson's disease. Mov. Disord. 24, 1641-1649. doi: 10.1002/mds.22643

Beck, A. T., Steer, R. A., and Brown, G. K. (1996). BDI-II, Beck depression inventory : Manual. San Antonio, Tex.; Boston: Psychological Corp; Harcourt Brace. New York, NY: Springer.

Becker, S., Baumer, A., Maetzler, W., Nussbaum, S., Timmers, M., Van Nueten, L., et al. (2020). Assessment of cognitive-driven activity of daily living impairment in non-demented Parkinson's patients. J. Neuropsychol. 14, 69-84. doi: 10.1111/ jnp. 12173

Beyle, A., Glonnegger, H., Cerff, B., Graber, S., Berg, D., and Liepelt-Scarfone, I. (2018). The Multiple Object Test as a performance-based tool to assess the decline of ADL function in Parkinson's disease. PLoS One 13:e0200990. doi: 10.1371/journal.pone.0200990

Bhimani, R. (2014). Understanding the Burden on Caregivers of People with Parkinson's:a Scoping Review of the Literature. Rehabil. Res. Pract. 2014:718527. doi: 10.1155/2014/718527

Bronnick, K., Ehrt, U., Emre, M., De Deyn, P. P., Wesnes, K., Tekin, S., et al. (2006). Attentional deficits affect activities of daily living in dementia-associated with Parkinson's disease. J. Neurol. Neurosurg. Psychiatry 77, 1136-1142. doi: 10.1136/jnnp.2006.093146

Brown, R. G., MacCarthy, B., Jahanshahi, M., and Marsden, C. D. (1989). Accuracy of self-reported disability in patients with parkinsonism. Arch. Neurol. 46, 955-959. doi: 10.1001/archneur.1989.00520450025014

Caap-Ahlgren, M., and Dehlin, O. (2002). Factors of importance to the caregiver burden experienced by family caregivers of Parkinson's disease patients. Aging Clin. Exp. Res. 14, 371-377. doi: 10.1007/BF03324464

Cahn-Weiner, D. A., Farias, S. T., Julian, L., Harvey, D. J., Kramer, J. H., Reed, B. R., et al. (2007). Cognitive and neuroimaging predictors of instrumental activities of daily living. J. Int. Neuropsychol. Soc. 13, 747-757. doi: 10.1017/ S1355617707070853

Camargo, C. H. F., Bronzini, A., Tolentino, E. S., Medyk, C., and Schultz-Pereira, G. L. (2018). Can the CERAD neuropsychological battery be used to assess cognitive impairment in Parkinson's disease? Arq. Neuropsiquiatr. 76, 145-149. doi: 10.1590/0004-282x20180003

Cernera, S., Eisinger, R. S., Wong, J. K., Ho, K. W. D., Lopes, J. L., To, K., et al. (2020). Long-term Parkinson's disease quality of life after staged DBS: STN vs GPi and first vs second lead. NPJ Park. Dis. 6:13. doi: 10.1038/s41531-0200115-3

Chaudhuri, K. R., Martinez-Martin, P., Schapira, A. H., Stocchi, F., Sethi, K., Odin, P., et al. (2006). International multicenter pilot study of the first comprehensive self-completed nonmotor symptoms questionnaire for Parkinson's disease: the NMSQuest study. Mov. Disord. 21, 916-923. doi: 10.1002/mds.20844

Chen, Y. S., Chen, H. L., Lu, C. H., Lee, C. Y., Chou, K. H., Chen, M. H., et al. (2021). The corticolimbic structural covariance network as an early predictive biosignature for cognitive impairment in Parkinson's disease. Sci. Rep. 11:862. doi: 10.1038/s41598-020-79403-x

Cheon, S. M., Park, K. W., and Kim, J. W. (2015). Identification of Daily Activity Impairments in the Diagnosis of Parkinson Disease Dementia. Cogn. Behav. Neurol. 28, 220-228. doi: 10.1097/WNN.0000000000000081

Cholerton, B., Poston, K. L., Tian, L., Quinn, J. F., Chung, K. A., Hiller, A. L., et al. (2020). Participant and Study Partner Reported Impact of Cognition on Functional Activities in Parkinson's Disease. Mov. Disord. Clin. Pract. 7, 61-69. doi: $10.1002 / \mathrm{mdc} 3.12870$

Christ, J. B., Fruhmann Berger, M., Riedl, E., Prakash, D., Csoti, I., Molt, W., et al. (2013). How precise are activities of daily living scales for the diagnosis of Parkinson's disease dementia? A pilot study. Park. Relat. Disord. 19, 371-374. doi: 10.1016/j.parkreldis.2012.11.004

Copeland, J. N., Lieberman, A., Oravivattanakul, S., and Troster, A. I. (2016). Accuracy of Patient and Care Partner Identification of Cognitive Impairments in Parkinson's Disease-Mild Cognitive Impairment. Mov. Disord. 31, 693-698. doi: $10.1002 / \mathrm{mds} .26619$
Deck, B. L., Xie, S. X., Choi, G., Rick, J., Siderowf, A., Rudovsky, S., et al. (2019). Cognitive Functional Abilities in Parkinson's Disease:agreement Between Patients and Informants. Mov. Disord. Clin. Pract. 6, 440-445. doi: 10.1002/ mdc3.12781

Dubois, B., Burn, D., Goetz, C., Aarsland, D., Brown, R. G., Broe, G. A., et al. (2007) Diagnostic procedures for Parkinson's disease dementia: recommendations from the movement disorder society task force. Mov. Disord. 22, 2314-2324. doi: $10.1002 / \mathrm{mds} .21844$

Emre, M., Aarsland, D., Brown, R., Burn, D. J., Duyckaerts, C., Mizuno, Y., et al. (2007). Clinical diagnostic criteria for dementia associated with Parkinson's disease. Mov. Disord. 22, 1689-1707. doi: 10.1002/mds.21507

Farias, S. T., Lau, K., Harvey, D., Denny, K. G., Barba, C., and Mefford, A. N. (2017) Early Functional Limitations in Cognitively Normal Older Adults Predict Diagnostic Conversion to Mild Cognitive Impairment. J. Am. Geriatr. Soc. 65, 1152-1158. doi: 10.1111/jgs.14835

Fellows, R. P., and Schmitter-Edgecombe, M. (2019). Multimethod assessment of everyday functioning and memory abilities in Parkinson's disease. Neuropsychology 33, 169-177. doi: 10.1037/neu0000505

Fengler, S., Kessler, J., Timmermann, L., Zapf, A., Elben, S., Wojtecki, L., et al. (2016). Screening for Cognitive Impairment in Parkinson's Disease: improving the Diagnostic Utility of the MoCA through Subtest Weighting. PLoS One 11:e0159318. doi: 10.1371/journal.pone.0159318

Foster, E. R., and Doty, T. (2021). Cognitive Correlates of Instrumental Activities of Daily Living Performance in Parkinson Disease Without Dementia. Arch. Rehabil. Res. Clin. Transl. 3:100138. doi: 10.1016/j.arrct.2021.100138

Foster, E. R., and Hershey, T. (2011). Everyday Executive Function Is Associated With Activity Participation in Parkinson Disease Without Dementia. OTJR 31, 16-22. doi: 10.3928/15394492-20101108-04

Goetz, C. G., Tilley, B. C., Shaftman, S. R., Stebbins, G. T., Fahn, S., MartinezMartin, P., et al. (2008). Movement Disorder Society-sponsored revision of the Unified Parkinson's Disease Rating Scale (MDS-UPDRS): scale presentation and clinimetric testing results. Mov. Disord. 23, 2129-2170. doi: 10.1002/mds. 22340

Gorecka-Mazur, A., Furgala, A., Krygowska-Wajs, A., Pietraszko, W., Kwinta, B. and Gil, K. (2019). Activities of Daily Living and Their Relationship to HealthRelated Quality of Life in Patients with Parkinson Disease After Subthalamic Nucleus Deep Brain Stimulation. World Neurosurg. 125, e552-e562. doi: 10. 1016/j.wneu.2019.01.132

Harris, P. A., Taylor, R., Thielke, R., Payne, J., Gonzalez, N., and Conde, J. G. (2009). Research electronic data capture (REDCap)-a metadata-driven methodology and workflow process for providing translational research informatics support. J. Biomed. Inform. 42, 377-381. doi: 10.1016/j.jbi.2008.08.010

Hindmarch, I., Lehfeld, H., de Jongh, P., and Erzigkeit, H. (1998). The Bayer Activities of Daily Living Scale (B-ADL). Dement. Geriatr. Cogn. Disord. 9, 20-26. doi: 10.1159/000051195

Hobson, J. P., Edwards, N. I., and Meara, R. J. (2001). The Parkinson's Disease Activities of Daily Living Scale: a new simple and brief subjective measure of disability in Parkinson's disease. Clin. Rehabil. 15, 241-246. doi: 10.1191/ 026921501666767060

Hosking, A., Hommel, A., Lorenzl, S., Coelho, M., Ferreira, J. J., Meissner, W. G., et al. (2021). Characteristics of Patients with Late-Stage Parkinsonism Who are Nursing Home Residents Compared with those Living at Home. J. Am. Med. Dir. Assoc. 22, 440-445.e2. doi: 10.1016/j.jamda.2020.06.025

Kalampokini, S., Hommel, A., Lorenzl, S., Ferreira, J. J., Meissner, W. G., Odin, P., et al. (2020). Caregiver Burden in Late-Stage Parkinsonism and Its Associations J. Geriatr. Psychiatry. Neurol. 35, 110-120. doi: 10.1177/0891988720968263

Kalbe, E., Rehberg, S. P., Heber, I., Kronenbuerger, M., Schulz, J. B., Storch, A., et al. (2016). Subtypes of mild cognitive impairment in patients with Parkinson's disease: evidence from the LANDSCAPE study. J. Neurol. Neurosurg. Psychiatry 87, 1099-1105. doi: 10.1136/jnnp-2016-313838

Karrasch, M., Laatu, S., Martikainen, K., and Marttila, R. (2013). CERAD test performance and cognitive impairment in Parkinson's disease. Acta Neurol. Scand. 128, 409-413. doi: 10.1111/ane.12138

Landis, J. R., and Koch, G. G. (1977). The measurement of observer agreement for categorical data. Biometrics 33, 159-174. doi: 10.2307/2529310

Leritz, E., Loftis, C., Crucian, G., Friedman, W., and Bowers, D. (2004). Selfawareness of deficits in Parkinson disease. Clin. Neuropsychol. 18, 352-361. doi: 10.1080/1385404049052412 
Liepelt-Scarfone, I., Fruhmann Berger, M., Prakash, D., Csoti, I., Graber, S., Maetzler, W., et al. (2013). Clinical characteristics with an impact on ADL functions of PD patients with cognitive impairment indicative of dementia. PLoS One 8:e82902. doi: 10.1371/journal.pone.0082902

Liepelt-Scarfone, I., Graber, S., Kalbe, E., Riedel, O., Ringendahl, H., Schmidt, N., et al. (2021). [Guidelines for the Neuropsychological Assessment of Patients with Parkinson's Disease]. Fortschr. Neurol. Psychiatr. 89, 363-373. doi: 10. 1055/a-1099-9332

Lin, A., Brook, J., Grill, J. D., and Teng, E. (2017). Participant-Informant Relationships Affect Quality of Life Ratings in Incipient and Clinical Alzheimer Disease. Am. J. Geriatric. Psychiatry 25, 297-307. doi: 10.1016/j.jagp.2016.10. 007

Litvan, I., Goldman, J. G., Troster, A. I., Schmand, B. A., Weintraub, D., Petersen, R. C., et al. (2012). Diagnostic criteria for mild cognitive impairment in Parkinson's disease: movement Disorder Society Task Force guidelines. Mov. Disord. 27, 349-356. doi: 10.1002/mds.24893

Martinez-Martin, P., Benito-Leon, J., Alonso, F., Catalan, M. J., Pondal, M., Tobias, A., et al. (2003). Patients', doctors', and caregivers' assessment of disability using the UPDRS-ADL section: are these ratings interchangeable? Mov. Disord. 18, 985-992. doi: 10.1002/mds.10479

Medijainen, K., Paasuke, M., Lukmann, A., and Taba, P. (2015). Functional Performance and Associations between Performance Tests and Neurological Assessment Differ in Men and Women with Parkinson's Disease. Behav. Neurol. 2015:519801. doi: 10.1155/2015/519801

Morris, J. C., Mohs, R. C., Rogers, H., Fillenbaum, G., and Heyman, A. (1988). Consortium to establish a registry for Alzheimer's disease (CERAD) clinical and neuropsychological assessment of Alzheimer's disease. Psychopharmacol. Bull. 24, 641-652.

Mosley, P. E., Moodie, R., and Dissanayaka, N. (2017). Caregiver Burden in Parkinson Disease: a Critical Review of Recent Literature. J. Geriatr. Psychiatry. Neurol. 30, 235-252. doi: 10.1177/0891988717720302

Nasreddine, Z. S., Phillips, N. A., Bedirian, V., Charbonneau, S., Whitehead, V., Collin, I., et al. (2005). The Montreal Cognitive Assessment, MoCA: a brief screening tool for mild cognitive impairment. J. Am. Geriatr. Soc. 53, 695-699. doi: 10.1111/j.1532-5415.2005.53221.x

Pennington, C., Duncan, G., and Ritchie, C. (2021). Altered awareness of cognitive and neuropsychiatric symptoms in Parkinson's disease and Dementia with Lewy Bodies:a systematic review. Int. J. Geriatr. Psychiatry 36, 15-30. doi: 10.1002/ gps.5415

Pfeffer, R. I., Kurosaki, T. T., Harrah, C. H. Jr., Chance, J. M., and Filos, S. (1982). Measurement of functional activities in older adults in the community. J. Gerontol. 37, 323-329. doi: 10.1093/geronj/37.3.323

Pirogovsky, E., Schiehser, D. M., Obtera, K. M., Burke, M. M., Lessig, S. L., Song, D. D., et al. (2014). Instrumental activities of daily living are impaired in Parkinson's disease patients with mild cognitive impairment. Neuropsychology 28, 229-237. doi: 10.1037/neu0000045

Pusswald, G., Wiesbauer, P., Pirker, W., Novak, K., Foki, T., and Lehrner, J. (2019). Depression, quality of life, activities of daily living, and subjective memory after deep brain stimulation in Parkinson disease-A reliable change index analysis. Int. J. Geriatr. Psychiatry 34, 1698-1705. doi: 10.1002/gps.5184

Ransmayr, G. (2020). [Caregiver burden in patients with Parkinson's disease]. Fortschr. Neurol. Psychiatr. 88, 567-572. doi: 10.1055/a-1120-8567

Razani, J., Kakos, B., Orieta-Barbalace, C., Wong, J. T., Casas, R., Lu, P., et al. (2007). Predicting caregiver burden from daily functional abilities of patients with mild dementia. J. Am. Geriatrics. Soc. 55, 1415-1420. doi: 10.1111/j.15325415.2007.01307.x

Reijnders, J. S., Ehrt, U., Weber, W. E., Aarsland, D., and Leentjens, A. F. (2008). A systematic review of prevalence studies of depression in Parkinson's disease. Mov. Disord. 23, 183-189. doi: 10.1002/mds.21803
Sadek, J. R., Stricker, N., Adair, J. C., and Haaland, K. Y. (2011). Performancebased everyday functioning after stroke: relationship with IADL questionnaire and neurocognitive performance. J. Int. Neuropsychol. Soc. 17, 832-840. doi: $10.1017 /$ S1355617711000841

Santos Garcia, D., de Deus Fonticoba, T., Cores Bartolome, C., Naya Rios, L., Garcia Roca, L., Martinez Miro, C., et al. (2021). Predictors of Loss of Functional Independence in Parkinson's Disease:results from the COPPADIS Cohort at 2Year Follow-Up and Comparison with a Control Group. Diagnostics 11:1801. doi: 10.3390/diagnostics11101801

Seltzer, B., Vasterling, J. J., Mathias, C. W., and Brennan, A. (2001). Clinical and neuropsychological correlates of impaired awareness of deficits in Alzheimer disease and Parkinson disease: a comparative study. Neuropsychiatry Neuropsychol. Behav. Neurol. 14, 122-129.

Shulman, L. M., Pretzer-Aboff, I., Anderson, K. E., Stevenson, R., Vaughan, C. G., Gruber-Baldini, A. L., et al. (2006). Subjective report versus objective measurement of activities of daily living in Parkinson's disease. Mov. Disord. 21, 794-799. doi: 10.1002/mds.20803

Skinner, J. W., Lee, H. K., Roemmich, R. T., Amano, S., and Hass, C. J. (2015). Execution of Activities of Daily Living in Persons with Parkinson Disease. Med. Sci. Sports Exerc. 47, 1906-1912. doi: 10.1249/MSS.00000000000 00598

Sturm, W., Willmes, K., and Horn, W. (1993). Leistungsprüfsystem für 50-90jährige (LPS 50+). Göttingen: Hogrefe.

Sulzer, P., Baumer, A., Hoang, H. G., Becker, S., Lonneker, H. D., Graessel, E., et al. (2020). Assessment of Cognitive-Driven PerformanceBased Activities of Daily Living Dysfunction in Parkinson's Disease. J. Int. Neuropsychol. Soc. 26, 430-440. doi: 10.1017/S13556177190 01322

Tomlinson, C. L., Stowe, R., Patel, S., Rick, C., Gray, R., and Clarke, C. E. (2010). Systematic review of levodopa dose equivalency reporting in Parkinson's disease. Mov. Disord. 25, 2649-2653. doi: 10.1002/mds.23429

Yamawaki, R., Nankaku, M., Kusano, Y., Tajima, A., Ikeguchi, R., and Matsuda, S. (2020). Evaluation of Cognitive Function in Relation to Progression of Parkinson Disease. Am. J. Phys. Med. Rehabil. 99, 626-629. doi: 10.1097/PHM. 0000000000001385

Zanetti, O., Geroldi, C., Frisoni, G. B., Bianchetti, A., and Trabucchi, M. (1999). Contrasting results between caregiver's report and direct assessment of activities of daily living in patients affected by mild and very mild dementia:the contribution of the caregiver's personal characteristics. J. Am. Geriatrics Soc. 47, 196-202. doi: 10.1111/j.1532-5415.1999.tb 04578.x

Conflict of Interest: The authors declare that the research was conducted in the absence of any commercial or financial relationships that could be construed as a potential conflict of interest.

Publisher's Note: All claims expressed in this article are solely those of the authors and do not necessarily represent those of their affiliated organizations, or those of the publisher, the editors and the reviewers. Any product that may be evaluated in this article, or claim that may be made by its manufacturer, is not guaranteed or endorsed by the publisher.

Copyright (c) 2022 Becker, Solbrig, Michaelis, Faust, Brockmann and Liepelt-Scarfone. This is an open-access article distributed under the terms of the Creative Commons Attribution License (CC BY). The use, distribution or reproduction in other forums is permitted, provided the original author(s) and the copyright owner(s) are credited and that the original publication in this journal is cited, in accordance with accepted academic practice. No use, distribution or reproduction is permitted which does not comply with these terms. 